\title{
Editorial
}

By Alan L. Bisno, MD

\section{Shuffling Our Streptococcal Strategies: In-Hospital and Out}

Streptococcal pharyngitis is one of man's commonest maladies. Over the past half century or more, there has been intense interest in the epidemiology, diagnosis, and management of "strep throat" as well as in the pathogenesis of its non-suppurative sequels, acute rheumatic fever (ARF) and acute glomerulonephritis (AGN). Modern concepts of the appropriate management of streptococcal pharyngitis date from post World War II, when the efficacy of penicillin in prevention of ARF was convincingly demonstrated. Current recommendations, as restated within the past few months by the Committee on Rheumatic Fever and Bacterial Endocarditis of the American Heart Association, ${ }^{1}$ call for specific documentation of the diagnosis of streptococcal pharyngitis by throat culture followed by prompt antimicrobial therapy, preferably with penicillin, for no less than ten days. Although this management plan seems simple and straightforward, its application in practice has presented many difficulties.

The throat culture-treatment strategy is, of course, most effective in the highest risk patient population, namely school aged children. Sore throat occurring during the first two to three years of life is rarely streptococcal, and the same could be said of sore throat in adults, particularly those whose family or work situations do not place them in intimate contact with school children. This fact is well illustrated in the article by Dr. Chatrchai Watanakunakorn in this month's issue. He found a low culture positivity rate, only $6.2 \%$, among hospital employees with sore throat. This rate is in the range of the expected carrier rate in adults, and the majority of the cultures were not strongly positive. Moreover, there was no evidence of spread of streptococci from the throats of culture positive employees to hospital patients. Faced with these data, Dr. Watanakunakorn rightly concludes, based upon cost-benefit considerations, that throat cultures should be applied selectively to this low risk adult population. It seems reasonable that culturing should be more extensive among patients whose clinical presentations

From the Division of Infectious Diseases, Department of Medicine, University of Tennessee, Center for the Health Sciences, Memphis, Tennessee.

Address reprint requests to Alan L. Bisno, MD, University of Tennessee, Center for the Health Sciences, 956 Court Avenue, Room 3H16, Memphis, TN 38163. strongly suggest streptococcal pharyngitis or in the presence of an increased incidence of hospital-acquired streptococcal infections. The latter has become a rare occurrence, despite the time-honored pedigree of Streptococcus pyogenes as a nosocomial pathogen.

Controversy over appropriate management of strep throat, however, goes far beyond the hospital walls. What, after all, are our reasons for identifying and treating patients with strep throat? In past, the primary impetus was undoubtedly the prevention of ARF. (Data on preventability of AGN by treatment of the antecedent streptococcal infection are much weaker.) We live in an era, however, in which the incidence of ARF has plummeted to unprecedentedly low levels, at least in North America ${ }^{2}$ and western Europe, while the incidence of strep throat does not appear to have diminished appreciably. We do not know to what extent antibiotic therapy of strep throat (so called "primary prevention" of ARF) may have been responsible for the disappearance of ARF, but it is unlikely to be the whole story. In the first place, one-third or more of ARF cases occur after asymptomatic streptococcal infection. Moreover, the decline in ARF began prior to the antibiotic era.

A second reason for treating strep throat is to ameliorate the symptoms. A raft of recent articles ${ }^{3-5}$ have confirmed the older findings of the Warren Air Force Base investigative group that antibiotics do indeed decrease somewhat the acute toxicity associated with strep throat. On the other hand, unless treatment is started rather promptly, it is difficult to differentiate the improvement caused by antibiotics from that which occurs naturally during the course of this self-limited bacterial infection. Finally, treatment may serve to prevent the occurrence of local suppurative complications, but most clinicians nowadays would agree that such complications are neither terribly frequent nor extraordinarily difficult to manage should they occur.

Thus, the throat culture is advocated in 1985 as much to exclude the diagnosis of strep throat as it is to identify and treat patients acutely infected with this organism. In the face of the extraordinarily low risk of ARF which has now been reported from numerous areas of the US, there is little justification for blunderbuss penicillin treatment of all sore throats, the great majority of which are due to viruses and other non-streptococcal agents. Blatant overtreatment is apt to occur when clinicians use clinical 
impressions as the basis of treatment decisions, whereas the throat culture may be estimated to have a negative predictive value of greater than $95 \%$ in ruling out strep throat.

Unfortunately, even physicians who religiously employ throat cultures may not deliver optimal care. This was dramatically illustrated in a survey involving nearly $80 \%$ of Rhode Island's primary care physicians. ${ }^{6}$ These physicians estimated that one of every seven patients seen in the winter and one of every fourteen patients in the summer had a throat culture. Eighty seven percent of respondents prescribed antibiotic therapy before the culture results were known and, even more distressing, almost $40 \%$ continued therapy for a full ten days even in the face of a negative culture result. In such circumstances, the throat culture is merely adding unnecessary and unwelcome padding to our growing health care bill. Over $40 \%$ of Rhode Island physicians indicated that they often did not receive the throat culture results in time to influence therapeutic decisions. This problem may well be soluble with the appearance on the market of a number of commercial kits for direct detection for group A streptococcal antigen in throat swabs. ${ }^{7,8}$ These kits will enable the practitioner to have a definitive result within an hour or less. They appear to be highly specific but to have poor sensitivity for $1+$ positive throat cultures (less than ten colonies per plate). Given the low risk of ARF, failure to detect such weak cultures may not be critical. Indeed, many such cultures may be supposed to originate from asymptomatic pharyngeal carriers rather than from acutely infected patients.

Therapy of strep throat has remained relatively easy, because, unlike many other gram-positive and gramnegative cocci, group A streptococci have never developed resistance to penicillin. Nevertheless, treatment with oral penicillin has not been entirely effective in eradicating the organism from the pharynx; failure rates in the $15 \%$ range are not uncommon. Recently, unexpectedly high treatment failure rates have been reported with benzathine penicillin $G$ as well. ${ }^{9}$ For the most part, these failures have occurred in asymptomatic carriers ${ }^{10}$ and thus are of less immediate concern. The reasons for failure of antibiotics to eradicate group A streptococci from the pharynx in all instances have been much debated. Suggested explanations include: a) failure of antimicrobials to enter oropharyngeal secretions; b) ineffectiveness of antibiotics on relatively dormant "resting" organisms; $c$ ) inactivation of cell-wall active antibiotics by beta lactamases of normal oropharyngeal flora, ${ }^{11}$ and, more recently d) penicillin tolerance. ${ }^{12}$

Recent data indicate that streptococci can be eradicated from the pharynx in almost all patients by the addition of a few days of rifampin to the standard penicillin regimen. ${ }^{13,14}$ There are admittedly very few indications for such combination therapy in everyday medical practice. One can however envision selected clinical situations wherein, for medical or psychological reasons, pharyngeal eradication may be desirable in a stubbornly resistant streptococcal carrier or in a patient felt at particularly increased risk of ARF.

The lowered risk of ARF has led most authorities to deemphasize some of the more stringent techniques used in the past to stamp out every last pharyngeal streptococcus. Most consultants nowadays would not recommend routine reculturing of patients after treatment, routine cultures of asymptomatic family contacts, or community based programs of streptococcal eradication, such as have been utilized in past years. Moreover, with ARF incidence rates of less than one per 100,000 school children per year in many areas, the need for parenteral benzathine penicillin $G$ injections in treatment of strep throat is being down-played by academic streptococcologists, as it has been for several years by canny pediatricians and family practitioners.

Dr. Watanakunakorn's article is but one example of the way in which we are reassessing old streptococcal dogmas in the light of the current, highly appropriate emphasis on cost-benefit and risk-benefit ratios. In an era in which the incidence of rheumatic fever has declined dramatically, we are taking a fresh look at our epidemiologic assumptions, diagnostic modalities, and therapeutic options. The result should be more rational, and certainly more practical, approaches to the all-too-familiar problem of streptococcal sore throat.

\section{REFERENCES}

1. Shulman ST, Amren DP, Bisno AL, et al: Prevention of rheumatic fever. American Heart Association Committee report. Circulation 1984; 70:1118A-1122A.

2. Land MA, Bisno AL: Acute rheumatic fever, a vanishing disease in suburbia. JAMA 1983; 249:895-898.

3. Nelson JD: The effect of penicillin therapy on the symptoms and signs of streptococcal pharyngitis. Pedialr Infect Dis 1984; 3:10-13.

4. Hall CB, Breese BB: Does penicillin make Johnny's strep throat better? Pediatr Infect Dis 1984; 3:7-9.

5. Krober MS, Bass JW, Michels GN: Streptococcal pharyngitis: Placebo-controlled double-blind evaluation of clinical response to penicillin therapy. JAMA 1985; 253:1271-1274.

6. Holmberg SC, Faich GA: Streptococcal pharyngitis and acute rheumatic fever in Rhode Island. JAMA 1983; 250:2307-2312.

7. Gerber MA, Spadaccini LG, Wright LL, et al: Latex agglutination tests for rapid identification of group A streptococci directly from throat swabs. J Pediatr 1984; 105:702-705.

8. Berkowitz CD, Anthony BF, Kaplan EL, et al: A cooperative study of latex agglutination to identify group A streptococcal antigen in throat swabs of patients with acute pharyngitis. $J$ Pedialr, to be published.

9. Gastanaduy AS, Huwe BB, Kaplan EL, et al: Failure of penicillin to eradicate group A streptococci during an outbreak of pharyngitis. Lancet 1980; II:498-502.

10. Kaplan EL, Gastanaduy AS, Huwe BB: The role of the carrier in treatment failures after antibiotic therapy for group A streptococci in the upper respiratory tract. J Lab Clin Med 1981; 98:326-335.

11. Brook 1: The role of beta-lactamase-producing bacteria in the persistence of streptococcal tonsillar infection. Rev Infect Dis 1984; 6:601-607.

12. Kim KS, Kaplan EL: Demonstration of penicillin tolerance in group A streptococci. Abstracts of the IXth Lancefield International Symposium on Streptococci and Streptococcal Diseases, Lake Yamanaka, Japan. September 10-13, 1984. No. 158, p 166.

13. Chaudhary S, Bilinsky SA, Hennessy JL, et al: Penicillin $V$ and rifampin for the treatment of group A streptococcal pharyngitis: A randomized trial of 10 days penicillin vs 10 days penicillin with rifampin during the final 4 days of therapy. $J$ Pediatr 1985; 106:481-486.

14. Shulman ST, Tanz R, Willert C, et al: Rifampin treatment for group A streptococcal carriers. Abstracts of the IXth Lancefield International Symposium on Streptococci and Streptococcal Diseases, Lake Yamanaka, Japan. September 10-13, 1984. No. 154, p 162. 